\title{
The dependence of $\mathrm{Pi} 2$ waveforms on periodic velocity enhancements within bursty bulk flows
}

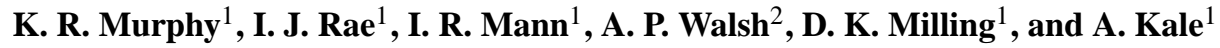 \\ ${ }^{1}$ Department of Physics, University of Alberta, Edmonton, Alberta, Canada \\ ${ }^{2}$ Mullard Space Science Laboratory, University College London, Dorking, UK
}

Received: 19 July 2010 - Revised: 15 February 2011 - Accepted: 16 February 2011 - Published: 10 March 2011

\begin{abstract}
Pi2s are a category of Ultra Low Frequency (ULF) waves associated with the onset of magnetic substorms. Recent work has suggested that the deceleration of bulk plasma flows in the central plasmasheet, known as bursty bulk flows (BBFs), are able to directly-drive Pi2 oscillations. Some of these studies have further shown evidence that there is a one-to-one correlation between Pi2 magnetic waveforms observed on the ground and periodic peaks in flow velocity within the BBF, known as flow bursts. Utilising a favourable conjunction between the Geotail spacecraft and the Canadian Array for Real-time Investigations of Magnetic Activity (CARISMA) magnetometer array on 31 May 1998, we examine the causality of the link between BBF flow bursts and Pi2 waveforms. Using a series of analytical tests in both the time and frequency domains, we find that while the $\mathrm{Pi} 2$ and BBF waveforms are very similar, the ground response for this event occurs prior to the observed flow enhancements in the magnetotail. We conclude that during this specific case study the temporal variations of the flow bursts within the $\mathrm{BBF}$ are not directly-driving the observed ground-based Pi2 waveforms, despite the fact that a visual inspection of both time-series might initially suggest that there is a causal relationship. We postulate that rather than there being a direct causal relation, the similar waveforms observed in both Pi2s and BBFs may result from temporal variations in a common source for both the BBFs and the Pi2s, such as magnetic reconnection in the tail, this source modulating both the $\mathrm{Pi} 2$ and $\mathrm{BBF}$ at the same frequency.
\end{abstract}

Keywords. Magnetospheric physics (Magnetosphereionosphere interactions; Magnetotail; Storms and substorms)

Correspondence to: K. R. Murphy

(kmurphy@phys.ualberta.ca)

\section{Introduction}

Impulsive Ultra Low Frequency (ULF) waves have been closely associated with the onset and development of magnetic storms and substorms for over 40 years (Jacobs et al., 1964). Historically, impulsive ULF waves known as Pi2s, with periods of $40-150 \mathrm{~s}$, have traditionally been used as indicators of substorm onset (e.g., Nose et al., 1998; Saito, 1969; Saito et al., 1976). In this paper we concentrate on the potential process or processes driving impulsive Pi2 ULF waves during substorms, a topic which is still being debated (e.g., Chi et al., 2001; Kepko and Kivelson, 1999; Keiling et al., 2006; Rae et al., 2006; Uozumi et al., 2000). In general it is believed that Pi2s are related to a disturbance in the near-Earth plasmasheet which subsequently leads to the formation of the substorm current wedge (SCW) (McPherron, 1979). The initial plasmasheet disturbance generates a fieldaligned current (FAC) system which closes in the ionosphere and enables substorm dipolarisation through the diversion of the cross-tail current system, established by the reflection of Alfvén waves from the ionosphere. These waves are seen as Pi2 waveforms superposed on magnetic bays observed on the ground (e.g., Olson, 1999, and references therein). A compressional disturbance may also propagate away from the source in the magnetotail and generate $\mathrm{Pi} 2$ phenomena in the ionosphere. These may include contributions to Pi2s in the form of field line resonances on the flanks, plasmaspheric cavity modes, as well as a contribution from the initial formation of the SCW (see e.g., Olson, 1999, and references therein).

The ULF waves responsible for the generation of the SCW FAC system are believed to be the dominant source of Pi2s observed at mid- to high-latitude magnetometer stations. In this scenario the initial FAC is established by an Alfvén wave which propagates to the ionosphere, and is reflected, perhaps multiple times. The observed ground-based magnetic perturbations are referred to as transient response (TR) Pi2s (Olson,

Published by Copernicus Publications on behalf of the European Geosciences Union. 
1999). If the plasmasheet disturbance is large enough, the existing cross-tail current is diverted along field lines into the ionosphere, and subsequently the SCW is observed as a large-scale night-side current system. Any impedance mismatch between the ionosphere and the current carrying Alfvén wave may then cause a partial reflection to occur, with the wave bouncing along field lines between the ionosphere and plasmasheet leading to the characteristic periodic and decaying structure of the Pi2 (Olson, 1999). However, Kepko et al. (2001) suggested that during periods of quiet geomagnetic activity the night-side ionospheric conductivity may be sufficiently low that any impedance mismatch between the ionosphere and Alfvén wave is negligible and produces little reflected ULF signal. Therefore during quiet periods the TR model could fail to adequately explain the characteristics of the observed Pi2 wavetrains.

The compressional, or fast-mode, wave produced by a substorm-related plasmasheet disturbance can travel almost isotropically. The compressional mode can couple to the plasmasphere forming a plasmaspheric cavity mode (e.g., Sutcliffe and Yumoto, 1991; Yeoman and Orr, 1989), or to an Alfvénic mode driving a field line resonance (e.g., Rae et al., 2006). The fast-mode wave is also believed to be the main source of Pi2s at higher latitudes when the TR mechanism is circumvented and the initial Alfvén wave does not strongly reflect at the ionosphere (Kepko et al., 2001). Uozumi et al. (2000), and Chi et al. (2001) proposed that the latitudinal amplitude and phase structure of Pi2s can be characterised by the flight path of a compressional disturbance in the equatorial plane of the magnetotail and an Alfvénic disturbance along the background magnetic field lines (cf. Tamao, 1964). Finally, Kepko and Kivelson (1999) suggested that Pi2s may be directly-driven by the deceleration of large amplitude earthward plasma flows, whilst Keiling et al. $(2006,2008)$ proposed that high-latitude Pi2 signals may directly represent the signatures of transient magnetotail reconnection, as well as a number of other Pi2 waveforms observed on the ground. Resolving the generation mechanism, or mechanisms, responsible for the generation of ground $\mathrm{Pi} 2$ signals is therefore of importance to fully understanding the magnetotail drivers of ground-based signatures.

Plasma flows in the central plasmasheet (CPS) due to steady convection typically have velocities of approximately $30 \mathrm{~km} \mathrm{~s}^{-1}$. However, high-speed flows are often observed with velocities approximately an order of magnitude larger than the typical plasmasheet velocity. These high-speed earthward flows are termed bursty bulk flows (BBFs) have typical velocities larger than $\sim 400 \mathrm{~km} \mathrm{~s}^{-1}$, and a duration of $\sim 10$ min (Angelopoulos et al., 1992). During these events short-lived, quasi-periodic, large amplitude peaks in plasma velocity can occur within a specific BBF, which are referred to as flow bursts (FBs) (Angelopoulos et al., 1994). BBFs are often observed as far as $\sim 30 R_{\mathrm{E}}$ down-tail and are rarely observed closer then $\sim 10 R_{\mathrm{E}}$ (e.g., Angelopoulos et al., 1992; Baumjohann et al., 1990). This is believed to be evidence of flow braking in the inner magnetosphere due to an increase in magnetic pressure caused by the increase in the strength of the Earth's magnetic field as flows propagate toward the Earth (e.g., Haerendel, 1992; Shiokawa et al., 1997).

Kepko and Kivelson (1999; also see Kepko et al., 2001) have suggested that high-speed earthward plasma flows may rapidly decelerate in the dipolar region of the Earth's magnetosphere and that the deceleration of temporal variations in the flow velocity are responsible for directly driving the Pi2 waveforms observed on the ground. By comparison of timeshifted FB earth-ward velocity time-series and Pi2 waveforms, Kepko and Kivelson (1999) and Kepko et al. (2001) suggested that for some substorm events the structure of ground-based Pi2 waveforms occurs as a direct consequence of each FB within a particular BBF. Specifically these authors suggested that additional FACs were induced inside the SCW region when BBFs are decelerated in the inner magnetosphere. This is termed the inertial current (IC) $\mathrm{Pi} 2$, and is hypothesised to be a small amplitude Pi2 waveform superposed onto the existing TR Pi2s and SCW. Further, these authors suggested that the deceleration of BBFs could additionally launch a fast-mode wave which perturbs magnetic field lines and directly drives Pi2s on the flanks and at lower latitudes, outside the SCW region.

We investigate the relationship between BBFs and Pi2 oscillations in the night-side magnetosphere by selecting a small night-side event during which plasma flows are observed in the magnetotail by the Geotail spacecraft when it is conjugate with the Canadian sector. A small localised ionospheric current system and magnetic bays are observed on the ground, which are consistent with a small substorm or pseudo-breakup. The Pi2 waveforms and BBF structure are shown to be remarkably similar; however, the ground signature is shown to occur prior to flow signatures in the CPS. We postulate that both signatures may be driven by the same source, which could allow for ground-based Pi2s to be observed prior to the $\mathrm{BBF}$ if magnetospheric conditions are favourable. Such a model is consistent with both the observations presented herein, and may also be consistent with those presented by Kepko and Kivelson (1999) and Kepko et al. (2001) as we discuss in this manuscript.

\section{Case study: 31 May 1998}

\subsection{In-situ observations}

In order to determine the relation between BBFs observed in the magnetotail and Pi2 oscillations on the ground we examined a conjunction between the Geotail satellite (Frank et al., 1994; Kokubun et al., 1994; Mukai et al., 1994) and the Canadian Array for Realtime Investigation of Magnetic Activity magnetometer array (Mann et al., 2008) (CARISMA, formally operated as CANOPUS, the Canadian Auroral Network for the OPEN Program Unified Study array (Rostoker 

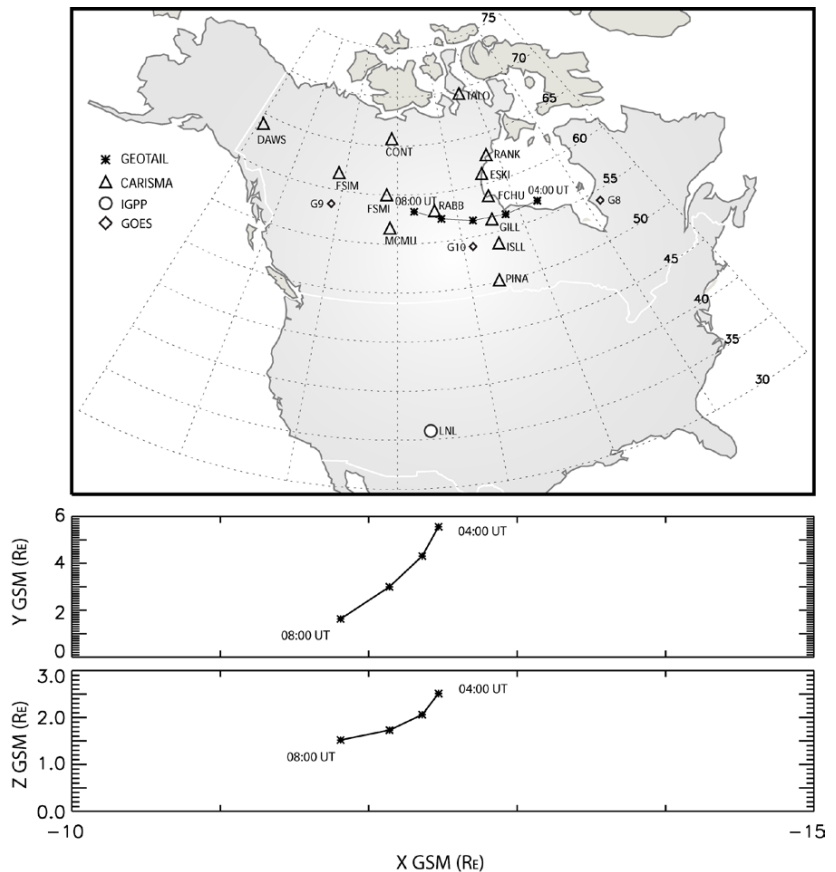

Fig. 1. (a) Ground northern magnetic field trace of the conjunction between the Geotail spacecraft and CARISMA magnetometer array between 04:00 and 08:00 UT on 31 May 1998. The north magnetic footprints of GOES-8, GOES-9 and GOES-10 are also shown. The GSM location of Geotail in the $x-y$ plane and $x-z$ planes are plotted in panels (b) and (c), respectively.

et al., 1995), prior to 1 April 2005). On 31 May 1998, a conjunction between the Geotail satellite and CARISMA occurred between 04:00 and 08:00 UT during which a small amplitude and localised night-side event was observed. The time propagated solar wind speed and proton number density during this conjunction, observed by WIND at $225 R_{\mathrm{E}}$ upstream, were $517 \mathrm{~km} \mathrm{~s}^{-1}$ and $1.8 \mathrm{n} / \mathrm{cc}$, respectively, while $B_{\mathrm{Z}}$ was southward and $B_{\mathrm{y}}$ was predominantly positive. During the conjunction the Geotail satellite was situated in the night-side CPS, and observed a short duration BBF during this conjunction. The top panel of Fig. 1 shows the northern magnetic footprint (obtained using Tsyganenko 89c external and IGRF internal geomagnetic fields (Tsyganenko, 1989), using the observed $\mathrm{Kp}=1$ ) of Geotail and the GOES8, GOES-9 and GOES-10 satellites along with the location of CARISMA and the lower latitude Los Alamos (LNL) magnetometers (see also Table 1). The middle and bottom panels show the location of Geotail in the GSM x-y and x-z planes, respectively.

During the conjunction between Geotail and CARISMA, a BBF was observed between 05:49-05:55 UT which contained four FB peaks. For the duration of the flow, Geotail was positioned at $\left(X_{\mathrm{GSM}}, Y_{\mathrm{GSM}}, Z_{\mathrm{GSM}}\right)=(-12.2,3.3$, 1.8) $R_{\mathrm{E}}$. The criterion that the ion-plasma beta be greater than 0.5 (Angelopoulos et al., 1992, 1994, and references

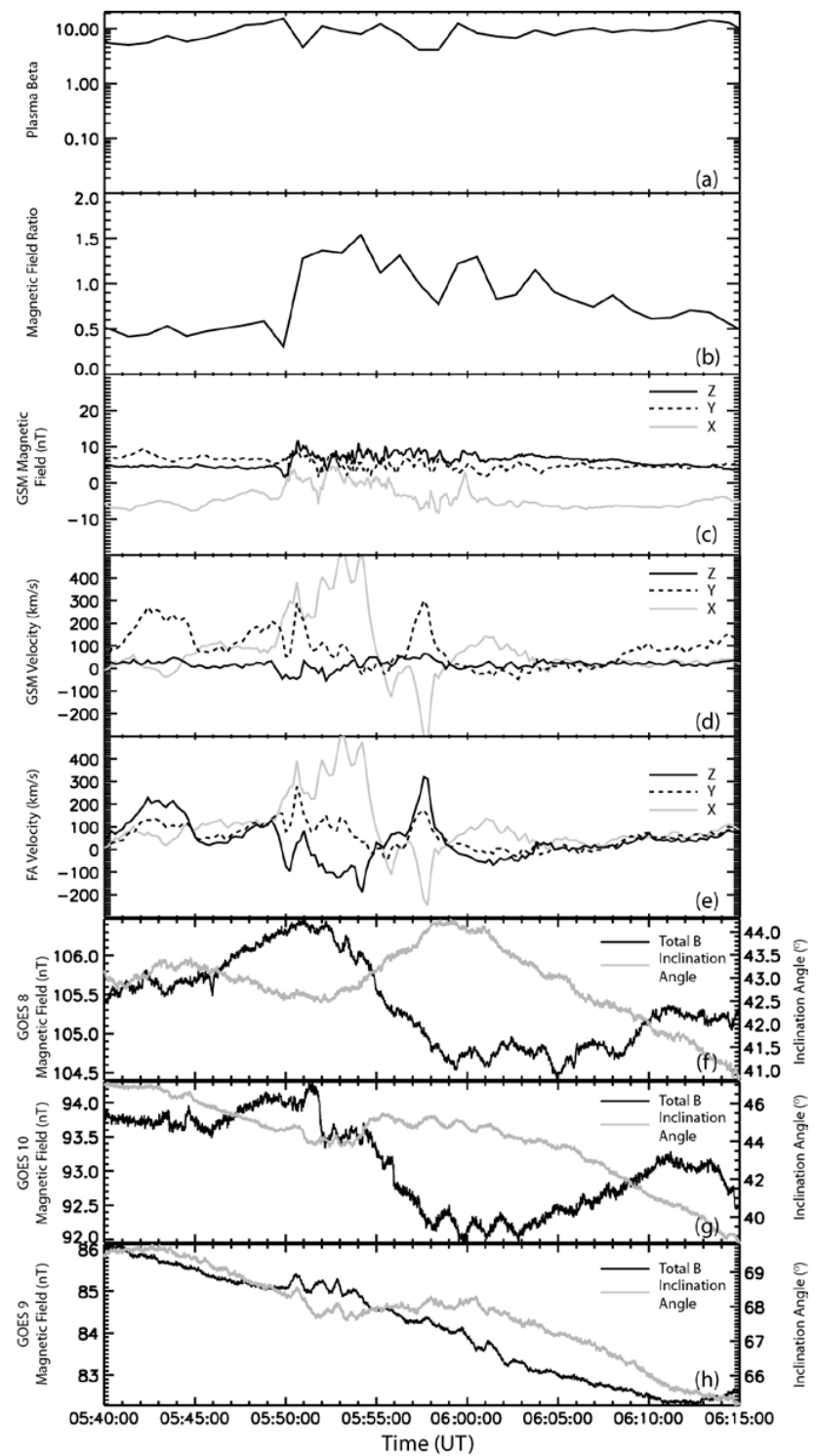

Fig. 2. In-situ and derived observations from Geotail for the period 05:45-06:15 UT. (a) Ion-plasma beta observed during the BBF. (b) The magnetic field ratio (left y-axis), the ratio of $B_{\mathrm{Z}}$ to vector sum of $B_{\mathrm{x}}$ and $B_{\mathrm{y}}$. (c) The GSM vector magnetic field. (d) The GSM plasma velocity and (e) plasma velocity in a field-aligned (FA) coordinate system. See text for details. (f)-(h) The total magnetic field and inclination angle (the angle between magnetic field vector and z-axis) at GOES-8, GOES-10 and GOES-9, respectively.

therein) is generally taken to indicate the spacecraft is situated within the CPS. Figure 2a confirms that Geotail is located in a region of hot plasma characteristic of the CPS for the duration of the interval as the derived ion-plasma beta lies well above the 0.5 threshold. The plasma beta was derived from measurements provided by the Comprehensive Plasma Instrument (CPI) at $64 \mathrm{~s}$ cadence (Frank et al., 1994) and the Geotail Magnetic Field Experiment (MGF) at $3 \mathrm{~s}$ cadence 
Table 1. Coordinates and L-shell values for the CARISMA and LNL magnetometers. All values were calculated using the International Geomagnetic reference Field for 1998.

\begin{tabular}{llcccccc}
\hline \multirow{2}{r}{ Station } & & \multicolumn{2}{c}{ Geographic (degrees) } & & \multicolumn{2}{c}{ Geomagnetic (degrees) } & L-Shell \\
Name & Code & Latitude & Longitude & & Latitude & Longitude & \\
\hline Taloyoak & TALO & 69.5 & 266.5 & & 79.0 & 328.6 & NA \\
Rankin Inlet & RANK & 62.8 & 267.9 & & 73.0 & 334.4 & 11.7 \\
Eskimo Point & ESKI & 61.1 & 266.0 & & 71.3 & 331.5 & 9.7 \\
Fort Churchill & FCHU & 58.8 & 265.9 & & 69.0 & 332.0 & 7.8 \\
Gillam & GILL & 56.4 & 265.4 & & 66.7 & 331.6 & 6.4 \\
Island Lake & ISLL & 53.9 & 265.3 & & 64.3 & 331.9 & 5.3 \\
Pinawa & PINA & 50.2 & 264.0 & & 60.6 & 330.4 & 4.2 \\
Rabbit Lake & RABB & 58.2 & 256.3 & & 67.4 & 317.3 & 6.8 \\
Contwoyto & CONT & 65.8 & 248.8 & & 73.3 & 302.2 & 12.1 \\
Fort Smith & FSMI & 60.3 & 248.1 & & 68.0 & 304.7 & 7.1 \\
Fort Mcmurray & MCMU & 56.7 & 248.8 & & 64.6 & 307.4 & 5.4 \\
Fort Simpson & FSIM & 61.8 & 238.8 & & 67.5 & 292.1 & 6.8 \\
Dawson & DAWS & 64.1 & 220.9 & & 65.9 & 271.9 & 6.0 \\
Los Alamos & LNL & 35.9 & 253.3 & & 44.4 & 318.4 & 2.0 \\
\hline
\end{tabular}

(Kokubun et al., 1994) down-sampled to match the cadence of the CPI instrument onboard the Geotail spacecraft.

Following the onset of the BBF, Geotail observes a dipolarisation of the GSM magnetic field evident in the increase of the ratio of the magnitude of $B_{\mathrm{z}}$ to the magnitude of the vector sum $B_{\mathrm{x}}$ and $B_{\mathrm{y}}$ (Fig. $2 \mathrm{~b}$ left-hand y-axis) and the zcomponent of the magnetic field (Fig. 2c, down-sampled to $64 \mathrm{~s}$ to match the cadence of the estimated plasma beta). The dipolarisation, observed at $\sim 05: 49: 30 \mathrm{UT}$, is characteristic of the relaxation of the magnetotail to a lower energy state during substorms, and is consistent with previous observations of BBFs during substorms (e.g., Angelopoulos et al., 1992). Note that during the interval of the BBF and FBs (05:4905:55 UT) no clear Pi2 pulsations were observed in the GSM magnetic field at Geotail (Fig. 2c) corresponding to the waveform observed in the FBs.

Panels (d) and (e) of Fig. 2 show the observed GSM plasma velocity at Geotail from the Low-Energy Particle (LEP) instrument at a $12 \mathrm{~s}$ cadence (Mukai et al., 1994) and the derived plasma velocity in field-aligned (FA) coordinates, respectively. The FA coordinate system is derived from a 20 min running mean of the GSM magnetic field. In the FA coordinate system the direction perpendicular to the background field and directed toward the earth is denoted by $x_{\mathrm{FA}}, z_{\mathrm{FA}}$ is aligned along the field and $y_{\mathrm{FA}}$ completes the right handed coordinate system (see also Rae et al., 2005). Clearly evident between 0549 UT and 0555 UT is a strong, $\sim 400 \mathrm{~km} \mathrm{~s}^{-1}$, earthward component of the perpendicular plasma velocity (hereafter referred to as $v_{\perp x}$ ), corresponding to a strong earthward BBF. During this interval four $\mathrm{FB}$ peaks are observed within the $v_{\perp x}$ component of the $\mathrm{BBF}$, with a temporal spacing on the order of one to two min- utes in the $\mathrm{Pi} 2$ period band and with velocity peaks between 400 and $500 \mathrm{~km} \mathrm{~s}^{-1}$. A detailed analysis of the 2-D ion distribution functions from the LEP Editor-B data (not shown) verifies that the plasma flow, BBF and FBs, observed at Geotail was indeed a bulk plasma flow perpendicular to the background field and not a field-aligned plasma beam (e.g., Raj et al., 2002). This further confirms that Geotail was likely situated in the CPS. Also apparent in Fig. 2 panel (d) is a large amplitude plasma flow $\left(\sim 250 \mathrm{~km} \mathrm{~s}^{-1}\right)$ in the $y_{\mathrm{GSM}}$ direction at approximately 05:40 UT. Note however, that when transformed into FA coordinates (Fig. 2e) this initial flow is predominantly directed anti-parallel to the background magnetic field $\left(z_{\mathrm{FA}}\right)$, has no earthward velocity component $\left(x_{\mathrm{FA}}\right)$, and further has no associated dipolarisation or fluctuation of the magnetic field (Fig. 2 panels b and c). Thus for this particular case study we concentrate on the perpendicular plasma flow $v_{\perp x}$ used to characterise the BBF which was observed to commence at 05:49 UT.

Figure 2 panels (f)-(h) show the magnitude of the magnetic field and angle of inclination (the angle between the $\mathrm{z}$ axis and magnetic field vector) observed at the three geosynchronous satellites GOES-8 (GOES-east), GOES-10 (closely conjugate to the ISLL magnetometer) and GOES-9 (GOESwest), respectively. Unlike Geotail the GOES satellites observe no dipolarisation or large amplitude deflection of the magnetic field typically associated with the formation of the SCW. During the conjunction of CARISMA and Geotail and observation of the BBF the observed magnetic field strength at all three geosynchronous satellites is approximately dipolar with magnitudes of $\sim 106,85$, and $96 \mathrm{nT}$ at GOES-8, GOES-9 and GOES-10 (respectively) and there is no large decrease in the angle of inclination typically associated with 


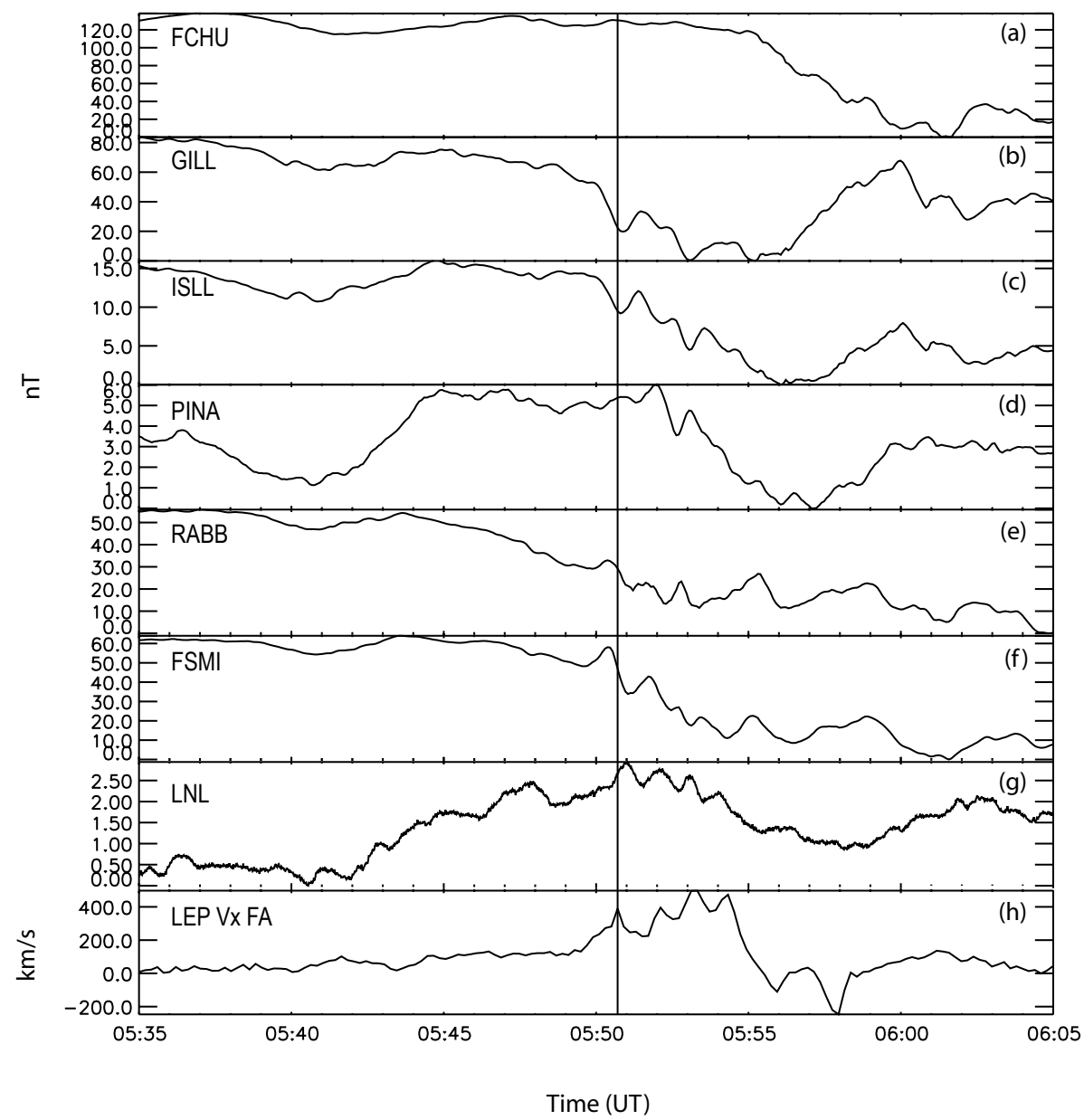

Fig. 3. A stack plot of the H-component magnetic field for selected CARISMA magnetometers and the Los Alamos (LNL) magnetometer, panels (a)-(g), and the $v_{\perp x}$ plasma flow observed by Geotail, panel (h), between 05:35-06:05 UT. The vertical line represents the time of maximum velocity of the first Geotail FB. Note that Pi2 pulsations at RABB and GILL are clearly evident before the initial flow burst.

a dipolarisation of the Earth's magnetic field. Moreover, similar to the magnetic field at Geotail, the GOES satellites observe little ULF wave activity during the interval of interest. Small amplitude oscillations on the order of half a nT are evident at $\sim 05: 50-05: 51$ UT at GOES 8 and 9; however, these pulsations occur later than the Pi2 pulsations observed on the ground and are not of the same frequency as the FBs within the BBF and Pi2s observed on the ground.

\subsection{Ground-based observations}

For the duration of the BBF, Geotail's footpoint was slightly to the west of the "Churchill line" of magnetometers ( $\sim 265$ degrees geographic longitude), and closely conjugate to the GILL and RABB magnetometers at geographic latitudes of 56.38, and 58.22 degrees, respectively (Fig. 1a and Table 1). For brevity, Fig. 3 shows the H-component of the magnetic field at the auroral- and mid-latitude Churchill line magnetometers (FCHU, GILL, ISLL, and PINA) as well as the stations RABB and FSMI and the low-latitude LNL magnetometer at a $5 \mathrm{~s}$ cadence. These stations represent those recording the largest magnetic bay structure, and are in closest conjunction with the Geotail satellite during the BBF interval, and so are the stations most likely to observe any IC Pi 2 driven by the deceleration of the BBF. The bottom panel of Fig. 3, (h), displays the $v_{\perp x}$ plasma flow measured by the LEP instrument at Geotail, the vertical line marks the time of maximum velocity within the initial FB peak.

Large amplitude magnetic deflections are observed at $L \approx$ 6.5 (GILL, RABB and FSMI), the largest bay being observed at FCHU $(\sim 120 \mathrm{nT})$. Though FCHU observes the largest $\mathrm{H}-$ component magnetic bay structure, the formation of the bay occurs much later than the observed BBF and thus is not considered in the time sequence of events related to the generation of Pi2's examined in the remainder of this manuscript. Conversely, the magnetic bays observed at GILL, RABB and FSMI begin to form at about 05:45 UT, evident in the weak deflection of the $\mathrm{H}$-component of the magnetic field, which 
begins approximately $4 \mathrm{~min}$ before the $\mathrm{BBF}$ is observed at Geotail. Any transient response (TR) $\mathrm{Pi} 2$ pulsations which might be associated with establishing the ionospheric currents responsible for the bays should be observed to begin during this initial deflection. However, the Pi2 wavepackets on the ground analysed herein are observed subsequent to the initial magnetic field deflection and nearly coincident in time with the BBF which is observed at 05:49 UT. As noted above, neither GOES-8, GOES-9 or GOES-10 observe any dipolarisation of the Earth's magnetic field typically resulting from the formation of a substorm current wedge and the onset of TR Pi2s, Fig. 2 panels (f)-(h). Hence, the Pi2s observed by the CARISMA and LNL magnetometers are unlikely to be TR Pi2 pulsations. This also suggests that the GILL, RABB and FSMI magnetometers map to a distance further away from the Earth than their nominal geosynchronous Lvalues (cf. Table 1).

Qualitatively, the vertical line in Fig. 3 guides the eye providing a method for visually estimating whether the $\mathrm{Pi} 2$ pulsations on the ground or the FBs in the CPS are observed first. At both the RABB and FSMI magnetometer stations it is clear that the impulsive Pi2 wavepacket occurs prior to the initial FB peak. However, at the other magnetometer stations it is more difficult to determine whether $\mathrm{Pi} 2$ pulsations on the ground or FBs in the CPS are observed first. For instance, at the ISLL and GILL magnetometers the deflection of the background magnetic field during the time of the BBF makes it difficult to visually identify the initial Pi2 impulse. Similarly, at LNL the relatively small amplitude of the $\mathrm{Pi} 2$ wavepacket also makes it difficult to isolate the start of the wavepacket from other oscillations. The following section presents a quantitative analysis of the relative timing between $\mathrm{Pi} 2$ pulsations observed on the ground and in the FBs in CPS to conclusively determine whether $\mathrm{Pi} 2$ pulsations on the ground or FBs in the CPS are observed first.

\subsection{Temporal causality of the $\mathrm{Pi}$ - $-\mathrm{BBF}$ waveforms}

Figure 3 clearly illustrates the onset of ground-based Pi2 pulsations closely coincident in time with the BBF observed by Geotail. However, to determine whether the BBF and Pi2 pulsations are in fact directly related (Kepko and Kivelson, 1999) we construct a series of tests to establish whether the $\mathrm{BBF}$ can directly be responsible for the $\mathrm{Pi} 2$ oscillations on the ground, establishing whether the $\mathrm{Pi} 2$ waveform is observed first in the BBF in the CPS and subsequently on the ground. By comparing the normalized dynamic power spectrum from the magnetometer stations to that of $v_{\perp x}$ we can determine whether $\mathrm{Pi} 2$ oscillations are observed first on the ground or in the magnetotail.

The dynamic power spectrum for both the magnetometers and the LEP $v_{\perp x}$ plasma flow is determined for the entire day using a 10 min raw time-series hanning windowed ( $\sim$ twice the temporal length of the BBF) with a 1 min step size (approximately the time spacing of individual FBs) The
CARISMA and LNL H-component and LEP $v_{\perp x}$ normalised dynamic power spectra are shown in Fig. 4a from 05:3006:15 UT. Note that each spectrum in the dynamic power spectra is time tagged with the middle time from the $10 \mathrm{~min}$ window used to calculate that particular spectrum.

Apparent in the LEP dynamic power spectra (bottom panel Fig. 4a) is a clear enhancement of $\mathrm{Pi} 2$ power between $\sim 05: 46-05: 47$ UT in the $11-17 \mathrm{mHz}$ range, highlighted by the black box, characterising the frequency content of FBs within the BBF. Similarly, the ground-based magnetometers each show an enhancement of $\mathrm{Pi} 2$ wave power in the $11-17 \mathrm{mHz}$ range as early as $\sim 05: 44 \mathrm{UT}$. Though both the ground-based magnetometer and LEP dynamic power spectra show a common enhancement of $\mathrm{Pi} 2$ wave power, to within $\sim 2$ min of each other, accurately determining whether this enhancement is in actuality observed first on the ground or in the CPS from the individual dynamic power spectra is difficult. However, by differencing the ground-based magnetometer dynamic power spectra and the Geotail LEP $v_{\perp x}$ spectra, we can determine whether Pi2 structures in the LEP FB $v_{\perp x}$ proceed or lag the $\mathrm{Pi} 2$ oscillations observed on the ground in a consistent and accurate manner. Figure $4 \mathrm{~b}$ shows the normalized dynamic power spectrum of the $v_{\perp x}$ plasma subtracted from the normalized dynamic power spectrum from each magnetometer, the resulting differenced power is plotted which we term a "dynamic difference power spectrum". If the BBF precedes (follows) the $\mathrm{Pi} 2$ we expect in each difference plot to first observe a minimum (maximum) in the spectra depicted by a blue (red) colour corresponding to a time where the $\mathrm{BBF}$ (ground $\mathrm{Pi}$ 2) power dominates, followed by a maximum (minimum) shown in red (blue) where ground Pi2 (BBF) power dominates. Figure $4 \mathrm{~b}$ shows the "dynamic difference power spectra" for the CARISMA (GILL, ISLL, PINA, RABB and FSMI) and LNL magnetometers between 05:30-06:15 UT. Note that in the relevant frequency range of study $(11-17 \mathrm{mHz}$, black box) power is observed first at the magnetometer stations (GILL, ISLL, PINA, RABB, FSMI and LNL - red), and only later does power from the BBF, $v_{\perp x}$, observed at Geotail dominate (blue). Consequently the data show that $\mathrm{Pi} 2$ power is seen on the ground in advance of being seen in the earthward plasma flow in the CPS at Geotail.

Additionally, we quantify the temporal causality between the BBF and Pi2 wavetrain via a lagged correlation and impulse response analysis, discussed below. Note however, these methods require the LEP $v_{\perp x}$ and ground-based magnetometer time-series to be narrow band filtered which may potentially introduce artificial signal and Gibbs effect phenomena to the time-series (Gibbs, 1898, 1899). In the following paragraphs we validate the use of a narrow band filter to isolate the $\mathrm{Pi} 2$ pulsations in both the ground-based magnetometer and LEP $v_{\perp x}$ time-series which are subsequently used for both a lagged correlation and impulse response analysis presented in the latter half of this section. 
(a) Dynamic Power Spectra
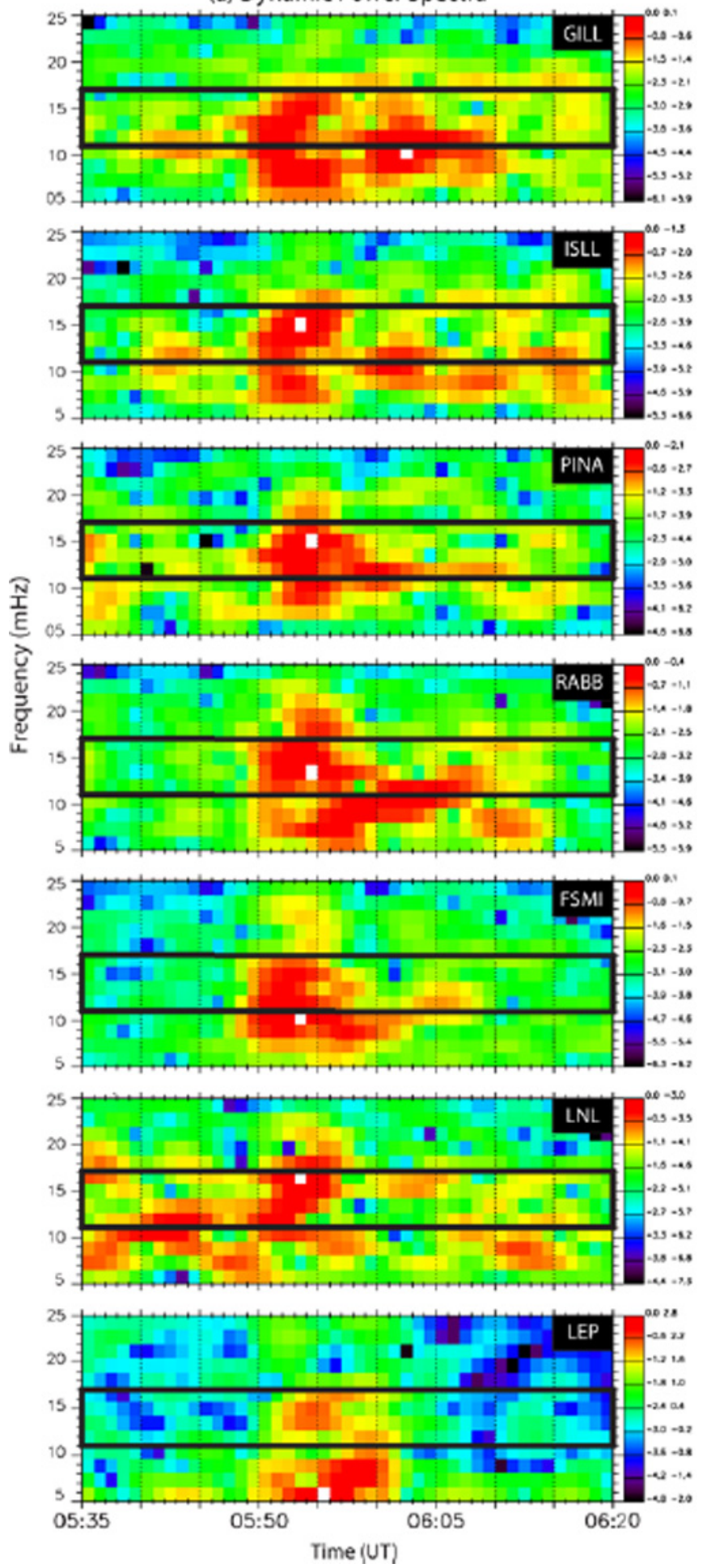

(b) Dynamic Difference Power Spectra
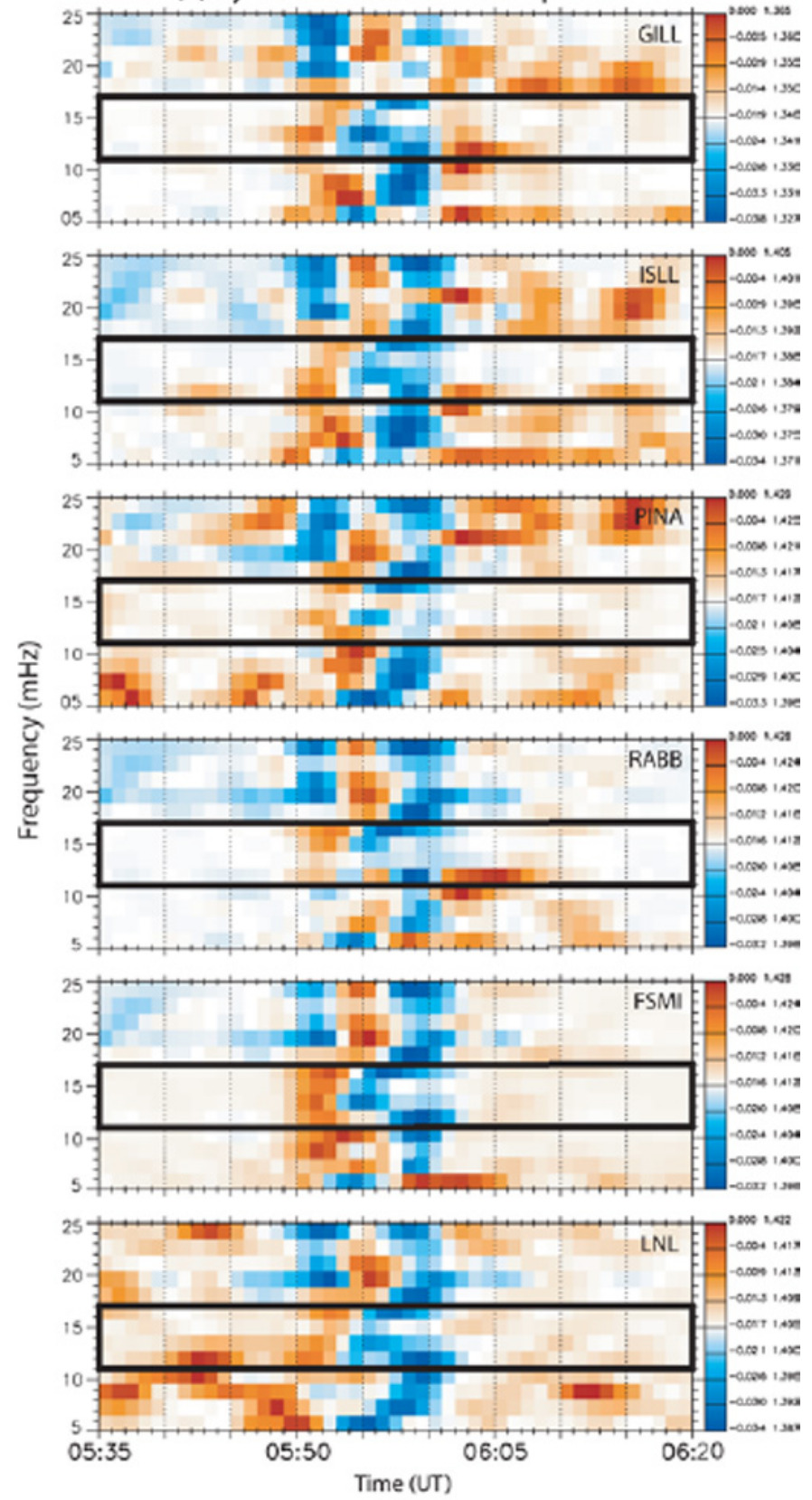

Fig. 4. (a) The H-component normalised dynamic power spectra at select CARSIMA magnetometers, the LNL magnetometer and the LEP $v_{\perp x}$ plasma flow. The color bar illustrates the normalised power at each station. (b) The dynamic difference spectra between the normalised power spectra in the H-component magnetic field at LNL, and selected CARISMA stations and the LEP $v_{\perp x}$ plasma flow. The color bar depicts the difference between the power observed at a ground-based magnetometer and that observed in $v_{\perp x}$. Red indicates power from the magnetometer dominates, blue shows that power in the LEP $v_{\perp x}$ plasma flow dominates and white shows similar power is observed in both the H-component magnetic field and $v_{\perp x}$ flow at Geotail. The black box in both (a) and (b) highlights the frequency band of interest, between $11-17 \mathrm{mHz}$. 
GILL
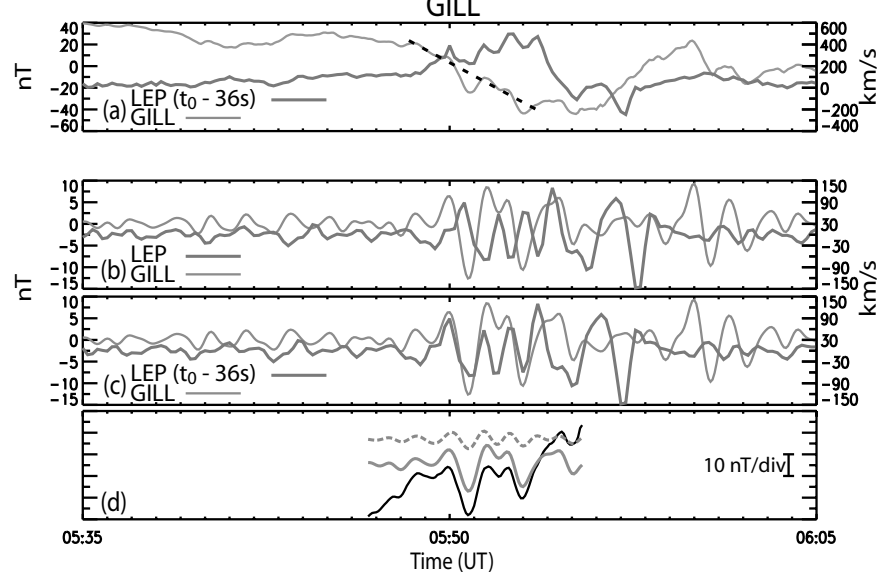

ISLL

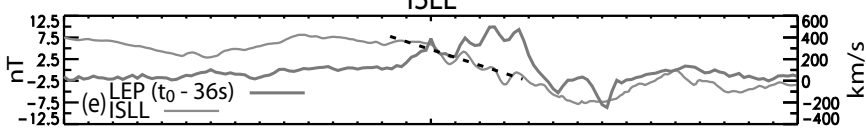

Fig. 5. (a) and (e) The GILL and ISLL, left and right respectively, raw magnetometer time-series. Overplotted in grey is the LEP $v_{\perp x}$ plasma flow shifted backward $36 \mathrm{~s}$ to the time of maximum correlation between the BBF waveform and magnetometer Pi2 pulsations The dashed grey line is a linear fit to the raw magnetometer time-series used to remove the background magnetic field fluctuations, see panels (d) and (h). (b) and (f) The Pi2 bandpass filtered (40-150 s) magnetometer (black) and LEP $v_{\perp x}$ plasma flow (grey). (c) and (g) The same as (b) however the LEP $v_{\perp x}$ plasma flow has been shifted 36s backward to show the high correlation between the BBF waveform and ground-based Pi2 pulsations. Panels (d) and (h) are a comparison of two methods for separating the magnetometer Pi2 pulsations from the background magnetic field. The black trace is a subtraction of the raw magnetometer data from a linear fit to the data, cf. panel (a). The two grey and dashed grey traces are the $40-150 \mathrm{~s}$ and $55-90 \mathrm{~s}$ bandpass filtered time-series. Note in panels (a) $-(\mathbf{b})$ and (e)-(g) the left hand axis is the magnitude of the magnetic field and the right is the magnitude of the plasma flow.

Table 2. Summarises the results at each magnetometer station for a maximum correlation lag with $v_{\zeta x}$ at Geotail.

\begin{tabular}{cccc}
\hline \multirow{2}{*}{$\begin{array}{c}\text { Magnetometer } \\
\text { station }\end{array}$} & $\begin{array}{c}\text { Maximum correlation } \\
\text { squared }\end{array}$ & \multicolumn{2}{c}{ Corresponding lag of BBF from $t_{0}(05: 49: 12 \mathrm{UT})$} \\
\cline { 3 - 4 } & 0.65 & -3 & Time Pts \\
GILL & 0.94 & -3 & -36 \\
ISLL & 0.76 & -6 & -36 \\
PINA & 0.74 & -7 & -72 \\
RABB & 0.37 & -8 & -84 \\
FSMI & 0.71 & -5 & -96 \\
LNL & & & -60 \\
\hline
\end{tabular}

Figure 5 panels (a) and (e), illustrate the raw magnetometer time-series (black) from GILL and ISLL, respectively, and the LEP $v_{\perp x}$ plasma flow time-series (grey) time-shifted backward $36 \mathrm{~s}$, which is the time of maximum correlation between the ground-based Pi2 pulsation and BBF (cf. Fig. 6 and Table 2). Note that the backward shift of the BBF suggests that $\mathrm{Pi} 2$ pulsations are observed first on the ground and then in the CPS (also observed in the dynamic difference power spectra cf. Fig. 4). Behrens and Glassmeier (1986) have suggested that TR Pi2 pulsations associated with the onset of magnetic substorms are a convolution of a driver function (the formation of the substorm bay) and a transfer function (the $\mathrm{Pi} 2$ pulsation) and that the deconvolution of the two signals is a more accurate method to separate the background signal from the Pi2 wavepacket than narrow band filtering. However it is clearly apparent in panels (a) and (e) of Fig. 5 that the time of maximum correlation between the BBF and Pi2 pulsations occurs subsequent to the initial deflection of the magnetic field at both GILL and ISLL. As discussed in the previous section this is strong evidence that the observed ground-based $\mathrm{Pi} 2$ pulsations are not the typical TR Pi2 pulsations related to the formation of the SCW and the initial deflection of the magnetic field. More importantly it suggests that bandpass filtering is adequate to separate the observed $\mathrm{Pi} 2$ pulsation from the background magnetic field fluctuations as the two phenomena appear to be decoupled and their onset separated in time in contrast to the results shown in Behrens and Glassmeier (1986).

Furthermore, spurious oscillations in filtered time-series are in general introduced at sharp discontinuities and at the edges of a signal window. In this particular case study the ground-based $\mathrm{Pi} 2$ pulsations are observed to follow the initial 


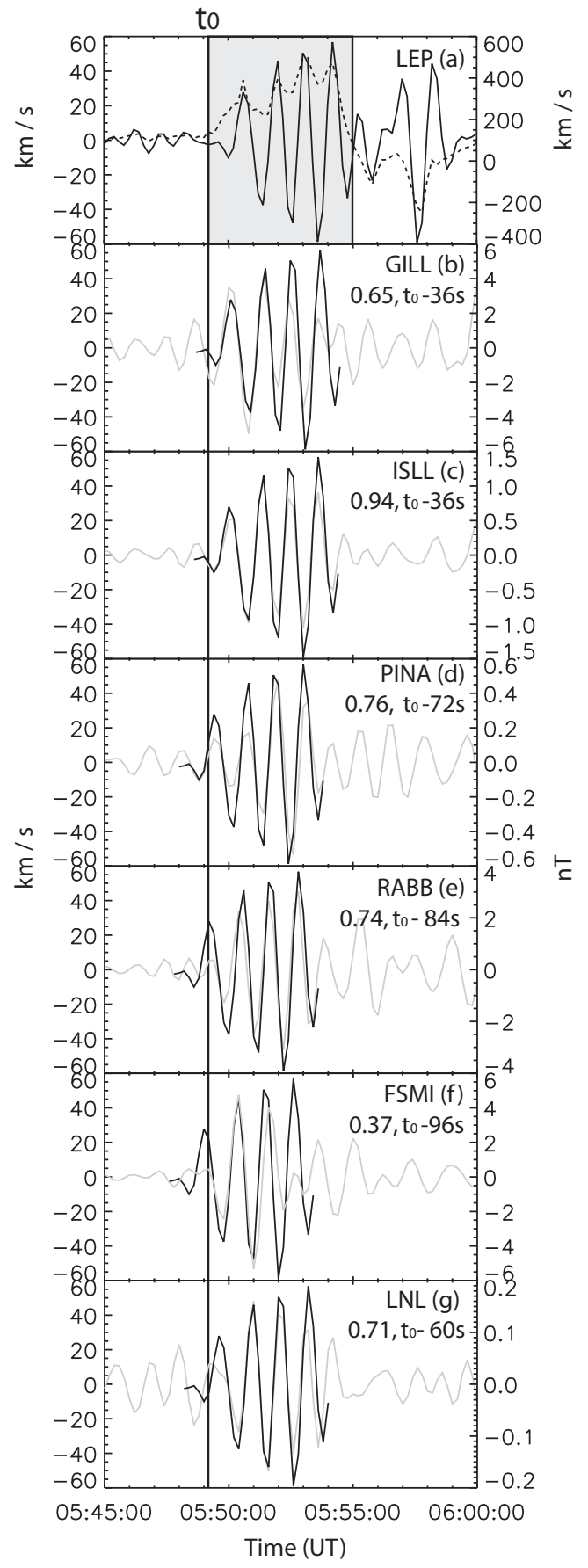

Fig. 6. The top panel, (a), shows the $v_{\perp x}$ plasma flow bandpass filtered between 55-95 s, approximately 11 and $17 \mathrm{mHz}$. The highlight depicts the section of the BBF which is used to calculate the BBF-Pi2 correlation coefficient; $t_{0}$ labels the beginning of the BBF, at 05:49:12 UT. Panels (b)-(g) show the Pi2 observed at each magnetometer station (grey), and over plotted is the BBF (black), this having been time-shifted such that the correlation between the ground magnetic pulsations and FBs is maximized. Displayed in the upper right corner of panels (b)-(g) is the correlation coefficient squared and the amount the BBF is shifted relative to $t_{0}$, cf., Table 2, e.g., $0.65, t_{0}-36 \mathrm{~s}$, in the case of the GILL magnetometer. and weak deflection of the magnetic field on the ground and are centred within the time-series being analysed far from the edges of the window. This limits the effect of Gibbs phenomena in our results due to narrow-band filtering. This is demonstrated in Fig. 5 panels (b)-(d) and (f)-(h). Panels (b) and (f) illustrate the Pi2 bandpass filtered (40-150 s) magnetometer (black) and LEP $v_{\perp} x$ (grey) time-series' utilising a Lanczos squared finite impulse response zero phase filter used in techniques such as complex demodulation (e.g. Beamish et al., 1979). Panels (c) and (g) are the same as (b) and (f), however the LEP $v_{\perp x}$ time-series has been shifted backward $36 \mathrm{~s}$ to the time of maximum correlation (see Fig. 6 and Table 2). Apparent in panels (b) and (f) is an approximate 180 degree phase shift between the $\mathrm{Pi} 2$ pulsations observed on the ground and the FBs. Panels (c) and (f) clearly show the BBF and $\mathrm{Pi} 2$ wavepacket are phase matched when the $\mathrm{BBF}$ is shifted back in time 36s. Comparing the raw time-series (a) and (e) as well as the $\mathrm{Pi} 2$ bandpass signals, panels (b), (c), (f) and (g) in Fig. 5, it is evident that the BBF is in fact correlated with the $\mathrm{Pi} 2$ wavepacket and not artificial oscillations introduced by bandpass filtering.

The bottom panels, (d) and (h), of Fig. 5 further demonstrate the robust nature of the bandpass Lanczos filter utilised in the lagged correlation and impulse response analysis. The black trace is a subtraction of a linear trend (illustrated in panel (a) and (e) by the thick dotted line) from the raw magnetometer data. The solid grey line and dashed grey line are the narrow band filtered ground-based time-series between $40-150 \mathrm{~s}$, the $\mathrm{Pi} 2$ waveband, and $55-90 \mathrm{~s}(\sim 11-17 \mathrm{mHz})$ characterising the periodicity of FBs within the BBF respectively (offset for clarity). Apparent in panels (d) and (h) is that no Gibbs phenomena is present when bandpass filtering. Note however, there is a decrease in the Pi2 amplitudes and it is possible to introduce a small phase shift in the bandpassed signal as a result of the width of the filter used . This is typical of any bandpass filtered time-series. Hence the subsequent bandpass filtering of the BBF and $\mathrm{Pi} 2$ signals between $55-90 \mathrm{~s}$ is adequate for studying the temporal causality of the $\mathrm{BBF}$ and Pi2 waveforms shown below.

A lagged correlation analysis between the BBF $v_{\perp x}$ and the observed ground $\mathrm{Pi} 2$ waveforms not only allows for the similarities between the Pi2 waveforms and BBF to be quantified but also provides a quantitative methodology to determine whether Pi2 wave power is observed first in the CPS or on ground. This allows for the causality of the BBF-Pi2 relationship to be directly analysed. It is important to note that when correlating the ground-based $\mathrm{Pi} 2$ and BBF signals both the phase and amplitude of the signals contribute to the correlation. In particular, the correlation will be maximised at a lag when the signals are both in phase and have similar amplitude profiles; the lag indicating whether ULF waves are observed first on the ground or a BBF is observed first in the CPS. Figure 6a shows the $v_{\perp x}$ plasma flow observed at the Geotail satellite filtered between $11-17 \mathrm{mHz}$ (solid) and the raw time-series (dashed). The highlighted 
area corresponds to the section of the $\mathrm{BBF}$ which is used to calculate the correlation between the FBs and $\mathrm{Pi} 2$ waveforms. Note that similar to panels (d) and (h) of Fig. 5, the top panel of Fig. 6 shows no evidence of the Gibbs phenomena in the filtered BBF time-series. Additionally, only a subsection of the BBF time-series is utilised (highlighted in grey) to ensure that only the FB waveforms are correlated with the ground-based magnetometer Pi2 signals. The minimum and maximum lags considered in the analysis correspond to $2 \mathrm{~min}$ (10 time steps either way of $t_{0}, 05: 49: 12 \mathrm{UT}$ ); these lags shift the BBF 2 min backward or forward of $t_{0}$, respectively. Figure 6, panels (b) $-(\mathrm{g})$ show the $11-17 \mathrm{mHz}$ filtered $\mathrm{Pi} 2 \mathrm{ob}-$ served at each ground station (grey). Over-plotted in black is the BBF $v_{\perp x}$ time-series, with a lag applied which generates the maximum squared correlation.

Table 2 summarises the results of the lagged correlation. A positive lag corresponds to the BBF occurring before the observation of ground Pi2s, and a negative lag implies that the Pi2 is observed before the BBF. Note that the correlations shown in Table 2 and Fig. 6 are the squared correlation coefficients. Apparent in Fig. 6 and Table 2 is that all magnetometer stations except for FSMI exhibit a high correlation with the BBF; however, the largest correlation occurs when the BBF is time-shifted backward (negative lag), suggesting that the $\mathrm{BBF}$ and $\mathrm{FBs}$ are not directly-driving the $\mathrm{Pi} 2$ pulsations observed on the ground. The correlation of the Pi2 pulsations observed in the D-component of the ground magnetic field (not shown) also suggests that the Pi2 pulsations on the ground precede the BBF flow in the CPS. However, the correlations in the D-component are in general smaller than those observed in the $\mathrm{H}$-component. It is interesting to note that the largest lag along the Churchill meridian is observed at the PINA magnetometer station, this is discussed in more detail in the next section.

It is important to note that the correlations we present in Table 2 and Fig. 6 represent the lags corresponding to the maximum correlation between the ground-based magnetometer Pi2 observations and the packet of BBF flow bursts. No visual analysis was used when determining the time shifts or lags. As can be seen in Fig. 3, for instance, a visual analysis of the BBF FBs and the LNL, RABB and FSMI magnetometer traces (Fig. 3) is perhaps suggestive that the initial FB may line up with the large amplitude Pi2 impulse rather than the small amplitude magnetic deflection identified in the correlation analysis (note, for example, that for GILL, ISLL and PINA the correlation analysis aligns the Pi2 with the initial FB). However, even if we consider the next largest value of the correlation, which occurs when the lag is translated forward by one wave period as might be expected by a visual analysis such that the initial large amplitude impulse at LNL, FSMI and RABB corresponds to the initial FB in the $\mathrm{BBF}$, the resultant lags are $0 \mathrm{~s},-24 \mathrm{~s}$ and $-24 \mathrm{~s}$, respectively. These represent shorter lags, but are still consistent with the ground-based Pi2s being observed before or at least coincident with the BBF in CPS.
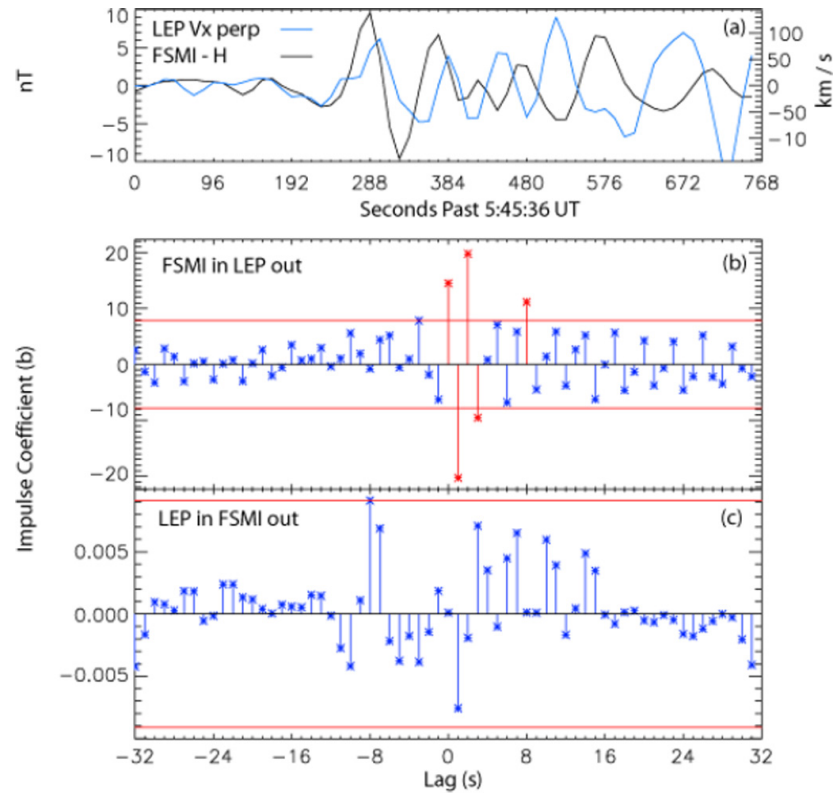

Fig. 7. Panel (a), shows time-series of the Pi2 observed at FSMI (black) in $\mathrm{nT}$ (left axis), and of the BBF (blue) in $\mathrm{km} \mathrm{s}^{-1}$ (right axis). These are the data sets utilised in the impulse response function. Panels (b) and (c) depict the results from the impulse response function, (b) BBF $v_{\perp x}$ as a function of the Pi2 waveform at FSMI and (c) the Pi2 waveform at FSMI as a function the BBF $v_{\perp x}$. The $\mathrm{y}$-axis is the value of the filter coefficient, $\beta$, as a function of the lag, $\mathrm{s}$, on the $\mathrm{x}$-axis. The horizontal lines represent the threshold defined in Eq. (2) and the red vertical lines represent the $\beta$ coefficients lying above the threshold.

Finally, the BBF-Pi2 causality may be rigorously tested utilising a lagged regression analysis in Fourier space, also known as an impulse response function (Shumway and Stoffer, 2006), shown in Eq. (1).

$y_{t}=\sum_{s=\frac{-N}{2}}^{\frac{N}{2}-1} \beta_{s} x_{t-s}+v_{t}$

The impulse response function utilises the Fourier spectrum of two signals, $x_{t}$ and $y_{t}$, to estimate a set of filter coefficients relating the two variables. Specifically, the impulse response method estimates the $\beta_{s}$ coefficients in Eq. (1), where $y_{t}$ and $x_{t}$ are two discrete signals and $v_{t}$ is a stationary noise process (e.g., a Gaussian distributed random signal), $N$ is the number of points in each time-series, $t$ is the time and $s$ is the lag between the two signals at time $t$. The filter coefficients $\beta_{s}$ are estimated from the power spectrum of $x$ and the crosspower of $x$ and $y$. Details on the estimation and calculation of each $\beta_{s}$ can be found in Shumway and Stoffer (2006).

There are two important properties of the impulse response function to note: If $s$ is a positive integer, then $y_{t}$ is a function of a series of past values of $x_{t}$. If $s$ is a negative value, then $y$ can be written in terms of future $\mathrm{x}$-values (or $x_{t}$ is a 
function of past values of $y_{t}$ ). By convention, $y$ is forced to be a function of the past values of $x_{t}$ by considering only the coefficients of $\beta_{s}$ where $s \geq 0$ and which satisfy the condition:

$\left|\beta_{s \geq 0}\right| \geq \max \left(\left|\beta_{s<0}\right|\right)$

The second property of the impulse response function is that Eq. (1) may be reversed and $x_{t}$ may be written as a function of $y_{t}$. In general one calculates both sets of $\beta_{s}\left(\beta_{s, y(x)}\right.$ and $\left.\beta_{s, x(y)}\right)$ and determines the most likely relation between the observed variables. Ideally, one set of $\beta_{s}$ will not satisfy Eq. (2) and the correct temporal dependence between $x$ and $y$ may be determined. Alternatively, neither sets of $\beta_{s}$ could satisfy Eq. (2) suggesting the variables are not causally related and are therefore independent, however similar their time-series appear to be. Finally, both sets of $\beta_{s}$ can satisfy Eq. (2) implying that there may be additional variables in the system relating $x$ and $y$ which have not been considered.

The impulse response function is therefore ideal to test the BBF-Pi2 relation as it directly relates the observed BBF to the Pi2 in a functional form. In our analysis we consider both the scenario where the $\mathrm{Pi} 2$ is a function of the $\mathrm{BBF}$ as well as the BBF as a function of the ground-based Pi2 pulsations. The first case suggests that the $\mathrm{BBF}$ and the $\mathrm{Pi} 2$ pulsations are directly related and that the observed Pi2 pulsations may be the IC Pi2 (Kepko et al., 2001). The second case suggests that another physical process may exist which modulates both the Pi2 and BBF at the same frequency but also that they are not causally related.

Figure 7 summarises the results from the impulse response analysis between the H-component magnetic field observed at the FSMI magnetometer and the $v_{\perp x}$ plasma velocity. Both datasets have been bandpass filtered in the frequency range of the BBF (55-95 s, $11-17 \mathrm{mHz}$ ); these bandpass signals are shown in the top panel of Fig. 7. The bottom two panels, (b) and (c), display the $\beta$ coefficients as a function of the lag $s$. The horizontal lines denote the maximum value of $\left|\beta_{s}\right|$ where $s<0$, and the vertical lines represent the value of $\beta$ at each $s$. The red vertical lines depict those values $\beta_{s}$ which satisfy Eq. (2). Figure $7 \mathrm{~b}$ shows the filter coefficients when the $v_{\perp x}$ plasma flow is considered as a possible function of the Pi2 time-series observed at FSMI. Evident in panel (b) is that the coefficients at lags $s=0,1,2,3$, and 8 rise above the threshold and satisfy Eq. (2). Figure 7c shows the filter coefficients when the Pi2 observed at FSMI is considered as a function of the $v_{\perp x}$ plasma flow observed at Geotail. Evident in Fig. 7c is that none of the coefficients satisfy Eq. (2). Table 3 summarises the results from the impulse response function for the Pi2s observed at the five remaining magnetometer stations. These results indicate that the FB in $v_{\perp x}$ are likely not the cause of the Pi2s observed on the ground (panel c). However, the fact the FB $v_{\perp x}$ can be written as a lagged function of the FSMI H-component magnetometer data, panel (b), suggest that the time-series are related, perhaps by a common driver.
Table 3. Summarises the results from impulse response function analysis between each of the individual magnetometer $\mathrm{H}$ component time-series and the observed BBF $v_{\perp x}$.

\begin{tabular}{lcc}
\hline \multirow{2}{*}{$\begin{array}{l}\text { Magnetometer } \\
\text { station }\end{array}$} & $\begin{array}{c}\text { Number of } \beta_{s} \text { coefficients satisfying Eq. (2) } \\
\text { of the observed BBF }\left(x_{t}\right)\end{array}$ & $\begin{array}{c}\text { BBF }\left(x_{t}\right) \text { as a function } \\
\text { of the observed Pi2 }\left(y_{t}\right)\end{array}$ \\
\hline GILL & 0 & 2 \\
ISLL & 0 & 2 \\
PINA & 5 & 0 \\
RABB & 0 & 3 \\
FSMI & 0 & 5 \\
LNL & 1 & 2 \\
\hline
\end{tabular}

\section{Discussion}

In this paper we present results from a night-side conjunction between the Geotail spacecraft and the CARIMSA magnetometer array on 31 May 1998 during the development of a small and localised substorm-like event. Utilising observations of the plasma flow in the CPS from the Geotail-LEP instrument, and Pi2 waveforms from the CARISMA and from LNL magnetometers, we investigate the relationship between quasi-periodic FBs within a BBF and Pi2 waveforms observed on the ground.

Typically the dominant Pi2 pulsations on the night-side occur as a result of the formation of the substorm current wedge and the reflection of Alfvén waves from the ionosphere following the initial plasma sheet disturbance during substorm expansion phase onset (Olson, 1999). These Pi2 pulsations are referred to as transient response (TR) Pi2 pulsations. However, Kepko et al. (2001) have suggested that during periods of quiet geomagnetic activity the conductivity of the ionosphere could be sufficiently low such that there is little reflection of the Alfvén wave responsible for generating Pi2s observed on the ground, and hence that the TR mechanism (Olson, 1999) could be circumvented. During the initial formation of the magnetic bays observed on the ground in this event the Pi2s typically associated with the formation of the SCW are not observed. This suggests that either the TR Pi2s are damped or the TR mechanism is completely circumvented due to low conductivity of the ionosphere as suggested by Kepko et al. (2001). Following the formation of the initial bays and the intensification of the auroral electrojet, a quasi-periodic high-speed plasma flow as well as a packet of ground $\mathrm{Pi} 2$ pulsations are observed closely conjugate in time. Additionally, the geosynchronous magnetic field observed at GOES-8, GOES-9 and GOES-10 (Fig. 2f) is approximately dipolar, with magnitudes of $\sim 106,85$ and $96 \mathrm{nT}$ (respectively) while the magnetic field at GEOTAIL (at radial distance $\sim 13 R_{\mathrm{E}}$ ) is highly stretched with a magnitude of $\sim 10 \mathrm{nT}$. From this we infer that the flows observed at Geotail have not yet been decelerated as they are observed 
outside the likely region of flow braking (Shiokawa et al., 1997).

The impulse response function, "dynamic difference power spectra" and lagged correlation tests provide a framework to determine if there exists a potential causal one-to-one relationship between the observed BBF and ground-based Pi2 pulsations (Kepko and Kivelson, 1999; Kepko et al., 2001). Figure $4 \mathrm{~b}$ shows the temporal development of power in the Pi2 frequency range in the ground Pi2s and $v_{\perp x}$, and Fig. 6 shows the best lagged correlation between the observed Pi2s and the BBF $v_{\perp x}$ (summarised in Table 2). Table 3 summarises the results from the impulse response analysis between the BBF and Pi2s. Apparent in Fig. $4 \mathrm{~b}$ is that $\mathrm{Pi} 2$ wave power is observed first on the ground and subsequently in the plasma flow at Geotail. Similarly the correlation is maximised at each magnetometer when the BBF is shifted backward in time, and the impulse response function shows that at all stations, except PINA and LNL, that the BBF $v_{\perp x}$ waveform can be described in terms of the time series data from the ground Pi2s. All of these results are consistent with a scenario where the ground-based Pi2 oscillations precede the FB oscillation observed in the BBF plasma flow by the Geotail satellite. Since the BBF is likely observed outside the region of flow braking and the $\mathrm{Pi} 2$ pulsations on the ground are observed in advance of the FBs within the BBF it is unlikely that the observed $\mathrm{Pi} 2$ pulsations are the IC Pi2 pulsation described by Kepko et al. (2001).

However both PINA and LNL show some evidence that their Pi2s can be written as a function of the BBF $v_{\perp x}$ FB structure. Indeed, the Pi2 at LNL can be described as both the dependent $\left(y_{t}\right)$ and independent $\left(x_{t}\right)$ variable described by Eq. (1), suggesting that the BBF and Pi2 may indeed be related via additional variables not considered in the impulse response analysis. However, the dynamic difference power spectra at both PINA and LNL show the development of $\mathrm{Pi} 2$ power prior to the observation of BBF power in the 11$17 \mathrm{mHz}$ band. Similarly, the Pi2 at both LNL and PINA is best correlated with the $v_{\perp} x$ plasma flow when $v_{\perp} x$ is shifted $60 \mathrm{~s}$ and $72 \mathrm{~s}$ backward in time, respectively, corresponding to approximately one wave period.

It is clear from the observations and analysis presented here that the frequency, occurrence and structure of the BBF FBs and the Pi2 waveforms observed on the ground are very similar, suggesting that the two phenomena may be intimately connected. Recent work by Kepko and Kivelson (1999) and Kepko et al. (2001) has suggested that BBFs are able to directly-drive IC Pi2 pulsations on the ground. However, this is inconsistent with the observations presented here as the Pi2 pulsations are observed before the BBF (cf., Fig. 4b, 6, and 7 and Tables 1 and 2). Even if we consider the possibility that the BBF can propagate at angle with respect to midnight meridian it still remains unlikely that the directly driven model can account for the observations which we present here. For instance, in this particular case study the observed BBF had a non-zero azimuthal velocity directed toward dusk implying that the BBF might propagate such that its phase fronts are no longer orthogonal to the midnight meridian. If the BBF phase fronts are not orthogonal to the midnight meridian it is possible that the BBF front could run ahead towards the flanks, for instance over the RABB or FSMI magnetometers, generating Pi2s observed prior to the FBs observed by Geotail in the near midnight meridian. However, in order for this to work the BBF front must remain coherent across an azimuthal region extending at least $5.5 R_{\mathrm{E}}$. Recent work by Nakamura et al. (2004) using Cluster has demonstrated that the azimuthal scale size of a BBF is approximately $1.5-2 R_{\mathrm{E}}$ thus in our opinion it is unlikely that a $\mathrm{BBF}$ has the spatial coherency required in a directly driven model.

We propose, rather than a BBF directly driving groundbased $\mathrm{Pi} 2$ pulsations, that the two phenomena are related by a common source capable of generating both a BBF in the CPS as well as ULF waves which are observed in the ionosphere by ground-based magnetometers. In this scenario a common driver in the magnetotail could provide a mechanism which modulates both the Pi2 waveforms observed on the ground and forms the FB structure within a BBF at the same frequency. More importantly, in such a scenario , the $\mathrm{Pi} 2$ pulsations on the ground are related to the impact of a ULF wave front which is much more likely to be spatially coherent over an extended radial and azimuthal region (see for instance Mann et al., 2002, and Rae et al., 2005) than a $\mathrm{BBF}$, or indeed than the internal FB structure (see above discussion), the latter of which is required in the directly driven model.

A common source in the magnetotail could, for instance, be bursty/non-steady reconnection (Keiling et al., 2006, 2008) In such a scenario, bursty reconnection, potentially driven by an irregular inflow of ions into the NENL reconnection region, could produce both a propagating compressional disturbance as well as a bulk plasma flow burst with similar temporal structure. Each subsequent "burst" of reconnection could in turn generate an additional compressional disturbance and plasma flow. This could result in a periodic structure being observed in both the bulk plasma flow, i.e. FBs, and in a series of travelling compressional disturbances. As the compressional disturbances propagate toward the Earth, they could in turn couple to the background magnetic field creating the Alfvénic Pi2s observed by ground-based magnetometers (cf. the Tamao travel time concept, Tamao, 1964).

In this scenario the individual BBF FBs and fast-mode wave fronts could have the same temporal spacing, perhaps accounting for the high correlation between ground-based $\mathrm{Pi} 2$ waveforms and the series of FBs within a BBF. Moreover the travel time of the BBF and fast-mode is dependent on magnetospheric conditions (plasma density and magnetic field strength), and the fast-mode wave may travel at a faster group velocity than the observed BBF, or vice-versa. At midto high-latitudes, if the fast-mode group velocity is larger 


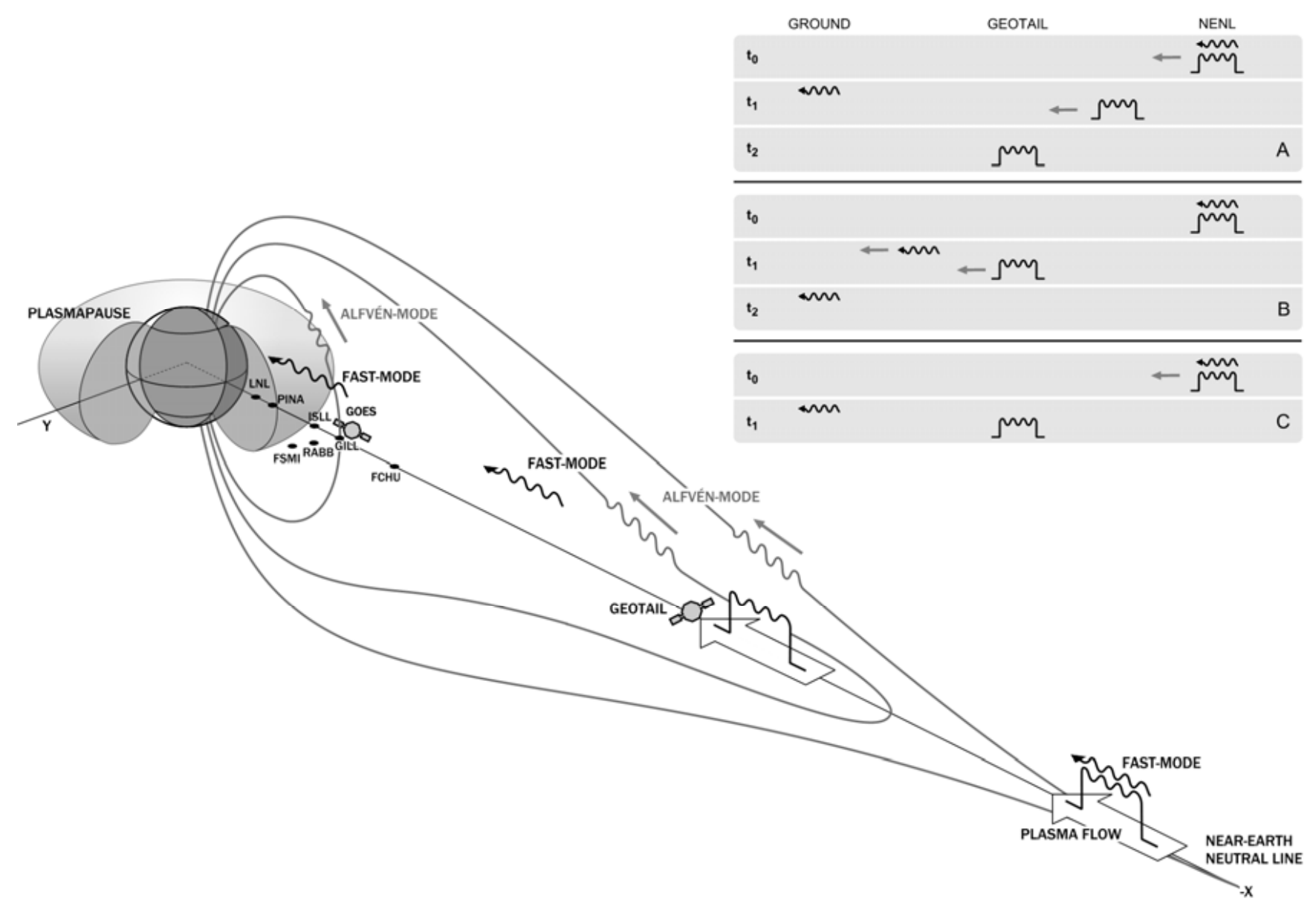

Fig. 8. An illustration depicting a potential sequence of events subsequent to reconnection at the NENL. Reconnection at the NENL results in the release of a BBF, and the production of an earthward propagating fast-mode which couples to the background field generating Pi2 pulsations observed on the ground. The inset panels (a)-(c) show three possible time lines, for $t_{0} \rightarrow t_{1} \rightarrow t_{2}$ of the relative times of observing a Pi2 pulsation on the ground and the in-situ observation of a BBF in the CPS. See text for details.

than the BBF flow speed in the CPS, it is possible that Pi2s could be observed on the ground before the BBF is observed in the magnetotail. This is consistent with the observation of Pi2 wavepackets observed on the ground ahead of the Geotail BFF during our event. Conversely, if the flow velocity exceeds the fast-mode group velocity, the BBF could be observed prior to the ground Pi2s. This is more consistent with the findings of Kepko and Kivelson (1999) and Kepko et al. (2001) who observed much greater BBF flow speeds than reported here. At low-latitudes (mapping to inside the plasmapause) the increase in density results in a decrease in both the fast-mode and Alfvén velocities, as well as an increase in the travel time from the initial tail source region to the ionosphere (e.g., Uozumi et al., 2000, and Chi et al., 2001). Thus at lower-latitudes, mapping close to and inside the plasmapause, it is possible for the ground Pi2s to be observed before, coincident with, or following the observation of the BBF flow in the tail. Figure 8 is a pictorial representation of the two mechanisms described above. The different propagation paths of the modulated signals are depicted in the inset panels (a)-(c) of Fig. 8. In case (a), the Pi2 is seen on the ground before the BBF FBs arrives at Geotail in the CPS; in case (b) the BBF FBs arrives at Geotail before the $\mathrm{Pi} 2$ is seen on the ground; and in case (c) the BBF FBs and the ground $\mathrm{P} i 2$ are seen at the same time.

Typical Alfvén velocities in the inner magnetosphere are between $\sim 4800 \mathrm{~km} \mathrm{~s}^{-1}$ outside of the plasmapause (e.g., Burton and Russell, 1970) and $\sim 490 \mathrm{~km} \mathrm{~s}^{-1}$ inside the plasmapause. Further down the tail, the Alfvén velocity can be much more variable; during the interval of the BBF the estimated Alfvén velocity in the CPS was $\sim 450 \mathrm{~km} \mathrm{~s}^{-1}$ (derived from local measurements at the Geotail satellite) whilst the Alfvén velocity in the distant tail and lobes can often exceed $1000 \mathrm{~km} \mathrm{~s}^{-1}$ (e.g., Burton and Russell, 1970). These velocities are consistent with the observations as well as the proposed BBF-Pi2 coupling mechanisms presented here. Initially, the fast-mode may have had a group velocity similar to that of the BBF. As the fast-mode propagates toward the 
Earth the magnetic field strength increases resulting in an increase of the fast-mode velocity. Assuming that the BBF and fast-mode are generated concurrently and that the BBF velocity remains either constant or is decelerated, then at highand mid- latitudes the Pi2 generated from the coupling of the fast-mode with the background magnetic field may be observed before the BBF in the mid-tail. At lower latitudes where the fast-mode and Alfvén velocities decrease, it is possible that the observation of the $\mathrm{BBF}$ and $\mathrm{Pi} 2$ are more closely coincident in time.

Interestingly, Keiling et al. (2006, 2008) presented case studies during differing nightside magnetotail driving conditions whereby favourable conjunctions between in-situ spacecraft between $8-18 R_{\mathrm{E}}$ were utilised to study the ground-based and in-situ signatures of Pi2s. In their studies, Keiling et al. $(2006,2008)$ found that there was a small time delay observed between the magnetic signature observed at Cluster situated in the nightside magnetosphere (16-18 $\left.R_{\mathrm{E}}\right)$ and mid-auroral latitude magnetometers $(L=4.5-6.1)$. That is, the in-situ magnetic signature of the Pi2 was observed $\sim 30$ s prior to that visually identified in the magnetometer time-series on the ground. Further, Keiling et al. (2008) demonstrated that there was, at times, a magnetic perturbation signature with an amplitude of $\sim 0.5 \mathrm{nT}$ observed by Geotail at $8-9 R_{\mathrm{E}}$. Taken together, these results were used to propose that a common source for ground-based and insitu Pi2s, specifically transient reconnection, which could account for both the short time delay between the signals as well as the similar frequency content of the Pi2 waveforms. Keiling et al. proposed that bursty reconnection generates a compressional disturbance which couples to the plasma sheet boundary layer generating Pi2 pulsations observed on the ground at mid- and auroral-latitudes. In their case studies, ground-based magnetometers at dipole L-shells between $L=4.5-6.1$ map close to the source region in the tail, i.e., close to the reconnection region in the tail.

In this study, we were able to estimate the location of the open-closed separatrix via visual identification of the cutoff in red-line auroral emissions using the CANOPUS GILL meridian scanning photometer (not shown; e.g., Blanchard et al., 1995; Rae et al., 2004), and find that this boundary resides close, but equatorward, of the FCHU station. The largest time lag (which characterises the shortest time of flight) between the in-situ BBF flow bursts and the Pi2 observed along the Churchill Line on the ground is at PINA, deep within the closed field line region, rather than occurring at a station that maps to the reconnection site as proposed by Keiling et al. (2006). Furthermore, we observed similar frequency content in the BBF flow burst and groundbased magnetic Pi2s, although unlike Keiling et al. we were unable to identify any definite evidence of in-situ magnetic Pi2 activity at Geotail (at $\sim 12 R_{\mathrm{E}}$ ). We do however observe small-amplitude magnetic Pi2 activity in the geosynchronous region. The propagation path for the fast-mode ULF wave fronts in the magnetotail may be complex. It is not clear whether they necessarily pass through the central plasmasheet, as in the direct propagation path outlined in a number of phenomenological models, including Tamao (1964), Kepko and Kivelson (1999) and Keiling et al. (2006). Future modelling of the wave coupling and dispersion in a 3-D magnetotail model would be valuable for supporting future studies.

While it is clear that the ULF wave propagation path is complex the relative timings along the meridian containing the GILL, ISLL, PINA and LNL magnetometers is similar to that of the Tamao travel path. Along this meridian the largest lag in the correlation analysis is observed at the PINA magnetometer station. At the lower latitude LNL station and higher latitude GILL and ISLL stations the lags are smaller suggesting that the Pi2 is observed first at the PINA magnetometer station. These results are quantitatively consistent with the time-of-flight model proposed by Tamao (1964, see also Uozumi et al., 2000, and Chi et al., 2001) who demonstrated that the fastest time-of-flight resided just outside of the plasmapause. If the PINA magnetometer station maps close to, but outside of, the plasmapause then we might expect a Pi2 to be observed first at the PINA magnetometer station (or have the largest lag) and subsequently at the other stations along the same meridian. Qualitatively our observations of the correlation of ground-based $\mathrm{Pi} 2$ pulsations with the FB structure within a BBF are consistent with the Tamao time-of-flight model as well as the phenomenological model described above. Though the relative time of flights are consistent with the Tamao (1964) travel path, more work is required to fully characterise the propagation path of a ULF wave front in the tail. Future work utilising the THEMIS mission could provide increased radial and azimuthal coverage of the magnetotail in order to better characterise the propagation path of ULF waves in the tail.

Finally, it is important to note that the scenario we have proposed is consistent with both the current disruption (CD) and NENL substorm paradigms. In the CD model, NENL reconnection, perhaps triggered by the arrival of a rarefaction wave, follows the initial expansion phase of the substorm which is driven by plasma instabilities in the near-Earth plasmasheet (e.g., Huba et al., 1977; Lui et al., 1995; Roux et al., 1991). The formation of the SCW in this CD model begins prior to reconnection at the NENL, and the generation of earthward propagating flows and fast-mode waves. In the NENL substorm model, reconnection initiates the substorm expansion phase. Flows in the magnetotail propagate toward the Earth disrupting the cross-tail current and diverting it into the ionosphere forming the SCW. In this scenario fast-flows in the CPS and the fast-mode launched at reconnection are observed prior to the formation of the SCW. In the CD paradigm, the Pi2s described by our model should be observed following the formation of the SCW and the observation of TR Pi2s. Conversely in the NENL model, the formation of the SCW, observation of TR Pi2s, and the $\mathrm{Pi} 2 \mathrm{~s}$ generated by the flows or fast-mode waves produced by 
reconnection should occur nearly simultaneously. This provides a verifiable time-line, during both substorm paradigms, with which to test our proposed model.

\section{Conclusion}

We present a night-side conjunction between the Geotail spacecraft and the CARISMA ground-based magnetometers on 31 May 1998 (04:00-08:00 UT), during a localised and small amplitude night-side activation which may be characterised as a small substorm or pseudo-breakup. During the Geotail-CARISMA conjunction, small amplitude Pi2 waves are observed by the CARISMA magnetometers and a highspeed plasma flow is observed at the Geotail satellite, in the form of a BBF. Recent work by Kepko and Kivelson (1999) and Kepko et al. (2001) has shown that there is a high correlation between $\mathrm{Pi} 2$ pulsations observed during substorms and the internal FB structure of BBFs. These authors have suggested that the deceleration of FBs, intermittent peaks in flow velocity within a BBF, generates a FAC which drives the $\mathrm{Pi} 2$ signatures observed on the ground, referred to as inertial current (IC) Pi2 pulsations. In their proposed model there is a one-to-one correlation between the multiple FBs and Pi2 wavetrain oscillations observed on the ground.

In this event the Geotail flow measurements and nightside Pi2 pulsations showed similar waveforms, with the Geotail measurements delayed relative to the ground $\mathrm{Pi} 2$. While the IC Pi2 described by Kepko et al. (2001) could explain the similarities between the Pi2 and BBF waveforms, the observed time delay between ground-based and in-situ observations of Pi2 waves and the BBF suggests the model is not appropriate in this case. We propose that the similarities between the observed $\mathrm{BBF}$ and $\mathrm{Pi} 2$ waveforms is a consequence of both phenomena being driven by a common source. Following bursts of NENL reconnection fast-mode waves may be excited which propagate Earthward coupling to the background magnetic field and exciting Alfvén waves which are observed on the ground as Pi2 pulsations. Depending on magnetospheric conditions, the ground $\mathrm{Pi} 2$ generated by the fast-mode could be observed before the BBF is seen in-situ in the CPS or vice-versa. A common driver could account for the observations presented herein and potentially also for those presented by Kepko and Kivelson (1999) and Kepko et al. (2001).

It is important to note that this study has concentrated upon the relative timing of magnetotail BBF flow bursts and ground-based Pi2s. More studies utilising multi-point ground and magnetospheric observations are required to fully determine the character of the BBF-Pi2 relation during pseudo-breakups and magnetic substorms, especially to characterise the propagation paths of the ULF wave fronts through the magnetotail and nightside magnetosphere. The THEMIS constellation of satellites, in combination with the GOES spacecraft and related ground-based instrumentation allows for a more extensive study of substorms, BBF's and ULF waves in the magnetosphere during substorm expansion phase onset. Such measurements, using extensive ground observations from both magnetometers and all sky cameras as well as multi-point THEMIS probe observations in the magnetotail (Angelopoulos, 2008), can be used to further test the validity of the model proposed here.

Acknowledgements. The authors would like to thank V. Angelopoulos and G. Rostoker for helpful discussions during the preparation of this manuscript. This work was partially funded by a Canadian NSERC PGS-M, PGS-D and Alberta Ingenuity scholarships awarded to KRM, a Canadian NSERC Discovery Grant to IRM. IJR and AK are funded by the Canadian Space Agency and APW by UK STFC grant number PP/E/001173/1. The operation of CARISMA (www.carisma.ca), part of the Canadian Geospace Monitoring Program, is funded by the CSA via a contract to the University of Alberta. Data regarding the position and magnetic mapping of the Geotail satellite, Geotail magnetic field (MGF) data and low energy particle (LEP) data, and Geotail comprehensive plasma instrument (CPI) data, were obtained from NASA via SSC Web, from T. Nagai and T. Mukai through DARTS at the Institute of Space and Astronautical Science (ISAS) in Japan and from B. Paterson at the University of Iowa, respectively. CANOPUS data, now operated as CARISMA, was obtained from the Canadian Space Science Data Portal at www.cssdp.ca. Data from the GOES satellites wave provided courtesy of Howard J. Singer at the National Oceanic and Atmospheric Administration (NOAA).

Topical Editor R. Nakamura thanks A. Keiling and another anonymous referee for their help in evaluating this paper.

\section{References}

Angelopoulos, V.: The THEMIS Mission, Space Sci. Rev., 141, 434, 2008.

Angelopoulos, V., Baumjohann, B., Kennel, C. F., Coroniti, F. V., Kivelson, M. G., Pellat, R., Walker, R. J., and Luhr, H.: Bursty Bulk Flows in the Inner Central Plasma Sheet, J. Geophys. Res., 97, 4027-4039, 1992.

Angelopoulos, V., Kennel, C. F., Coroniti, F. V., Pellat, R., Kivelson, M. G., Walker, R. J., Russell, C. T., Baumjohann, W., Feldman, W. C., and Gosling, J. T.: Statistical Characteristics of Bursty Bulk Flow Events, J. Geophys. Res., 99, 21257-21280, 1994.

Baumjohann, W., Paschmann, G., and Luehr, H.: Characteristics of high-speed ion flows in the plasma sheet, J. Geophys. Res., 95, 3801-3809, 1990.

Beamish, D., Hanson, H. W., and Webb, D. C.: Complex demodulation applied to Pi2 geomagnetic puslations, Geophys. J. R. Astr. Soc., 58, 471-493, 1979.

Behrens, J. and Glassmeier, K. H.: Deconvolution as a method for the separation of Pie pulsations from background field variations, J. Geophys., 59, 195-201, 1986.

Blanchard, G. T., Lyons, L. R., Samson, J. C., and Rich, F. J.: Locating the Polar Cap Boundary from Observations of $6300 \AA$ Auroral Emission, J. Geophys. Res., 100(A5), 78557862, doi:10.1029/94JA02631, 1995. 
Burton, R. K. and Russell, C. T.: The Alfvén Velocity in the Magnetosphere and Its Relationship to ELF Emission, J. Geophys. Res., 75, 5582-5586, 1970.

Chi, P. J., Russell, C. T., Raeder, J., Zesta, E., Yumoto, K., Kawano, H., Kitamura, K., Petrinec, S. M., Angelopoulos, V., Le, G., and Moldwin, M. B.: Propagation of the preliminary reverse impulse of sudden commencements to low latitudes, J. Geophys. Res., 106, 18857-18864, 2001.

Frank, L. A., Ackerson, K. L., Paterson, W. R., Lee, J. A., and English, M. R.: The Comprehensive Plasma Instrumentation (CPI) for the Geotail spacecraft, J. Geomag. Geoelect., 46, 23-37, 1994.

Gibbs, J. W.: Fourier's Series, Nature, 59, 200, 1898.

Gibbs, J. W.: Fourier's Series, Nature, 59, 606-606, 1899.

Haerendel, G.: Disruption, ballooning or auroral avalanche: On the cause of substorms, Proceeding of ICS1, 417-420, 1992.

Huba, J. D., Gladd, N. T., and Papadopoulos, K.: The lower-hybriddrift instability as a source of anomalous resistivity for magnetic field line reconnection, Geophys. Res. Lett., 4, 125-128, 1977.

Jacobs, J. A., Kato, Y., Matsushita, S., and Troitskaya, V. A.: Classification of Geomagnetic Micropulsations, J. Geophys. Res., 69, 180-181, 1964

Keiling, A., Fujimoto, M., Hasegawa, H., Honary, F., Sergeev, V., Semenov, V. S., Frey, H. U., Amm, O., Rème, H., Dandouras, I., and Lucek, E.: Association of $\mathrm{Pi} 2$ pulsations and pulsed reconnection: ground and Cluster observations in the tail lobe at $16 R_{\mathrm{E}}$, Ann. Geophys., 24, 3433-3449, doi:10.5194/angeo-243433-2006, 2006.

Keiling, A., Mozer, F. S., Rème, H., Dandouras, I., Lucek, E., Fujimoto, M., Hasegawa, H., and Reeves, G. D.: Periodic traveling compression regions during quiet geomagnetic conditions and their association with ground Pi2, Ann. Geophys., 26, 33413354, doi:10.5194/angeo-26-3341-2008, 2008.

Kepko, L. and Kivelson, M. G.: Generation of Pi2 pulsations by bursty bulk flows, J. Geophys. Res., 104, 25021-25034, 1999.

Kepko, L., Kivelson, M. G., and Yumoto, K.: Flow bursts, braking, and Pi2 pulsations, J. Geophys. Res., 106, 1903-1915, 2001.

Kokubun, S., Yamamoto, T., Mario, H. A., Hayashi, K., Shiokawa, K., and Kawano, H.: The GEOTAIL Magnetic Field Experiment, J. Geomag. Geoelect., 46, 7-21, 1994.

Lui, A. T. Y., Chang, C.-L., and Yoon, P. H.: Preliminary nonlocal analysis of cross-field current instability for substorm expansion onset, J. Geophys. Res., 100, 19147-19154, 1995.

Mann, I. R., Voronkov, I., Dunlop, M., Donovan, E., Yeoman, T. K., Milling, D. K., Wild, J., Kauristie, K., Amm, O., Bale, S. D., Balogh, A., Viljanen, A., and Opgenoorth, H. J.: Coordinated ground-based and Cluster observations of large amplitude global magnetospheric oscillations during a fast solar wind speed interval, Ann. Geophys., 20, 405-426, doi:10.5194/angeo-20-4052002, 2002

Mann, I. R., Milling, D. K., Rae, I. J., Ozeke, L. G., Kale, A., Kale, Z. C., Murphy, K. R., Parent, A., Usanova, M., Pahud, D. M., Lee, E.-A., Amalraj, V., Wallis, D. D., Angelopoulos, V., Glassmeier, K.-H., Russell, C. T., Auster, H.-U., and Singer, H. J.: The Upgraded CARISMA Magnetometer Array in the THEMIS Era, Space Sci. Rev., 141, 413-451, 2008.

McPherron, R. L.: Magnetospheric Substorms, Rev. Geophys. Space Phys., 17, 657-681, 1979.

Mukai, T., Machida, S., Saito, Y., Hirahara, M., Tersawa, T., Kaya,
N., Obara, T., Ejiri, M., and Nishida, A.: The low energy particle (LEP) experiment onboard the Geotail satellite, J. Geomag. Geoelect., 46, 669-692, 1994.

Nakamura, R., Baumjohann, W., Mouikis, C., Kistler, L. M., Runov, A., Volwerk, M., Asano, Y., Vörös, Z., Zhang, T. L., Klecker, B., Rème, H., and Balogh, A.: Spatial scale of high-speed flows in teh plasma sheet observed by Cluster, Geophys. Res. Lett., 31, L09804, doi:10.1029/2004GL019558, 2004.

Nose, M., Iyemori, T., Takeda, M., T., K., Milling, D. K., Orr, D., Singer, H. J., Wothington, E. W., and Sumitomo, N.: Automated detection of Pi 2 pulsations using wavelet analysis: 1. Method and application for substorm monitoring, Earth Planets Space, 50, 773-783, 1998

Olson, J. V.: Pi2 Pulsations and substorm onsets: A review, J. Geophys. Res., 104, 17499-17520, 1999.

Rae, I. J., Kabin, K., Rankin, R., Fenrich, F. R., Liu, W., Wanliss, J. A., Ridley, A. J., Gombosi, T. I., and De Zeeuw, D. L.: Comparison of photometer and global MHD determination of the open-closed field line boundary, J. Geophys. Res., 109, A01204, doi:10.1029/2003JA009968, 2004.

Rae, I. J., Donovan, E. F., Mann, I. R., Fenrich, F. R., Watt, C. E. J., Milling, D. K., Lester, M., Lavraud, B., Wild, J. A., Singer, H. J., Reme, H., and Balogh, A.: Evolution and characteristics of global Pc5 ULF waves during a high solar wind speed interval, J. Geophys. Res., 110, A12211, doi:12210.11029/12005JA011007, 2005.

Rae, I. J., Mann, I. R., Milling, D. K., Dent, Z. C., and Kale, A.: Pi2 pulsations: Field Line resonances of a Driven Response, Proceeding of ICS8, 253-258, 2006.

Raj, A., Phan, T., Lin, R. P., and Angelopoulos, V.: Wind survey of high-speed bulk flows and field-aligned beams in the near-Earth plasma sheet, J. Geophys. Res., 107, 1419, doi:10.1029/2001JA007547, 2002.

Rostoker, G., Samson, J. C., Creutzberg, F., Hughes, T. J., McDiarmid, D. R., McNamara, A. G., Vallance Jones, A., Wallis, D. D., and Coggger, L. L.: Canopus - A ground-based instrument array for remotes sensing the high latitude ionosphere during the ISTP/GGS program, Space Sci. Rev., 41, 743-760, 1995.

Roux, A., Perraut, S., Robert, P., Morane, A., Pedersen, A., Korth, A., Kremser, G., Aparicio, B., Rodgers, D., and Pellinen, R.: Plasma sheet instability related to the westward travelling surge, J. Geophys. Res., 96, 17697-17714, 1991.

Saito, T.: Geomagnetic Pulsations, Space Sci. Rev., 10, 319-412, 1969.

Saito, T., Sakurai, T., and Koyama, Y.: Mechanism of association between Pi2 pulsation and magnetospheric substorm, J. Atmos. Terr. Phys., 38, 1265-1277, 1976.

Shiokawa, K., Baumjohann, B., and Haerendel, G.: Braking of high-speed flows in the near-Earth tail, Geophys. Res. Lett., 24, 1179-1182, 1997.

Shumway, R. H. and Stoffer, D. S.: Time Series Analysis and Its Applications: Second Edition, Springer, New York, 2006.

Sutcliffe, P. R. and Yumoto, K.: On the Cavity Mode Nature of Low-Latitude Pi 2 Pulsations, J. Geophys. Res., 96, 1543-1551, 1991.

Tamao, T.: The structure of three-dimensional hydromagnetic waves in a uniform cold plasma, J. Geomag. Geoelectr., 18, 89114,1964

Tsyganenko, N. A.: A magnetospheric magnetic field model with a 
warped tail current sheet, Planet Space Sci., 37, 5-20, 1989.

Uozumi, T., Yumoto, K., Kawano, H., Yoshikawa, A., Olson, J. V., Solovyev, S. I., and Vershinin, E. F.: Characteristics of Energy Transfer of Pi 2 Magnetic Pulsations: Latitudinal Dependence, Geophys. Res. Lett., 27, 1619-1622, 2000.
Yeoman, T. K. and Orr, D.: Phase and spectral power of midlatitude Pi2 pulsations: Evidence for a plasmaspheric cavity resonance, Planet Space Sci., 37, 1367-1383, 1989. 